\title{
Dynamic Contrast-Enhanced MRI Reveals Unique Blood-Brain Barrier Permeability Characteristics in the Hippocampus in the Normal Brain
}

\author{
(D). Ivanidze, (D) M. Mackay, (D) A. Hoang, (D).M. Chi, (D) K. Cheng, (D) Aranow, (D)B. Volpe, (DB. Diamond, and (DP.C. Sanelli
}

\begin{abstract}
SUMMARY: We report a prospective dynamic contrast-enhanced MR imaging analysis of region-specific blood-brain barrier permeability in 5 healthy subjects. By means of standardized postprocessing and ROI sampling methods, the hippocampi revealed significantly elevated area under the dynamic contrast-enhanced curve and significantly increased blood-brain barrier permeability metrics (volume transfer constant and volume in the extravascular extracellular space) from model-based quantitation. These findings suggest unique blood-brain barrier permeability characteristics in the hippocampus, which are concordant with previous animal studies, potentially laying the groundwork for future studies assessing patient populations in which hippocampal pathology plays a role.
\end{abstract}

ABBREVIATIONS: BBBP = blood-brain barrier permeability; DCE = dynamic contrast-enhanced; $K^{\text {trans }}=$ volume transfer constant; $S L E=$ systemic lupus erythematosus; $\mathrm{VE}=$ volume in the extravascular extracellular space

$\mathbf{T}$ he utility of in vivo BBB permeability (BBBP) assessment using dynamic contrast-enhanced MR imaging has been demonstrated in a wide range of diseases, including cerebrovascular ischemia and Alzheimer disease. The extended Tofts algorithm describes a well-perfused 2-compartment model, allowing bidirectional transport of contrast between the intravascular space and extravascular extracellular space and quantitative assessment of BBBP parameters, including volume transfer constant $\left(K^{\text {trans }}\right)$ or intravascular spaceto-extravascular extracellular space flux per tissue volume, and volume in the extravascular extracellular space (VE).

In humans, region-specific BBBP data are limited and heterogeneous, given region-specific differences in microvascular architecture and in BBB-related protein expression profiles. ${ }^{1,2}$ The hippocampus exhibits unique anatomic and physiologic proper-

Received April 30, 2018; accepted after revision December 17.

From the Department of Radiology (J.I.), Weill Cornell Medicine, New York New York; The Center for Autoimmune, Musculoskeletal and Hematopoietic Diseases (M.M., C.A., B.D.), The Center for Health Innovations and Outcomes Research (P.C.S.), and The Center for Biomedical Science (B.V.), Feinstein Institute for Medical Research, Manhasset, New York; Department of Radiology (A.H., P.C.S.) and Imaging Clinical Effectiveness and Outcomes Research Program (P.C.S.), Northwell Health, New Hyde Park, New York; and Department of Radiology (J.M.C., K.C.) Donald and Barbara Zucker School of Medicine (M.M., A.H., J.M.C., K.C., C.A., B.V., B.D., P.C.S.), Hofstra/Northwell School of Medicine, Hempstead, New York.

This work was supported by "Anti-NMDA Receptor Antibodies in Adult Brain Dysfunction, and Fetal Brain Development"; Principal Investigator: Betty Diamond, MD; National Institutes of Health research grant IP01A1073693, August 2008 to July 2019. Please address correspondence to Jana Ivanidze, MD, PhD, Department of Radiology, Weill Cornell Medicine, 525 East 68th Street, New York, NY 10065; e-mail: jai9018@med.cornell.edu; @jana_ivanidze

- Indicates open access to non-subscribers at www.ajnr.org

http://dx.doi.org/10.3174/ajnr.A5962 ties $^{3}$ and unique perfusion characteristics with dual arterial blood supply from the anterior and posterior circulation. ${ }^{4}$ Recent human studies in systemic lupus erythematosus correlated cognitive decline with region-specific abnormal findings in the hippocampus with FDG-PET. ${ }^{5}$ Additionally, animal studies have revealed increased protein expression of CD36, matrix metalloproteinase-13, and osteopontin correlated with increased hippocampal BBBP, ${ }^{6}$ and dynamic contrast-enhanced (DCE)-MR imaging-derived baseline contrast enhancement curves were highest in the hippocampi in control subjects. ${ }^{7}$ The purpose of our study was to evaluate region-specific BBBP metrics in healthy human subjects.

\section{MATERIALS AND METHODS \\ Subject Cohort}

In this prospective institutional review board (Department of Radiology, Northwell Health)-approved study, 6 subjects underwent DCE-MR imaging and clinical and neuropsychological evaluations under an ongoing National Institutes of Health/National Institute of Allergy and Infectious Diseases protocol, 1PO1A1073693. Exclusion criteria were active or prior neuropsychiatric symptoms; use of antidepressant, antipsychotic, or anxiolytic drugs; or a history of excessive alcohol or illicit drug use.

\section{Data Acquisition}

All subjects underwent DCE-MR imaging on a 3T magnet (Siemens Prisma, Erlangen, Germany). MR imaging sequences included 3DT1WI $(0.9 \times 0.9 \times 0.9 \mathrm{~mm}$ resolution, $256 \times 256 \times 240$ matrix size, and 0.9-mm slice thickness), axial T2WI, FLAIR, and susceptibilityand diffusion-weighted imaging according to standard departmental 

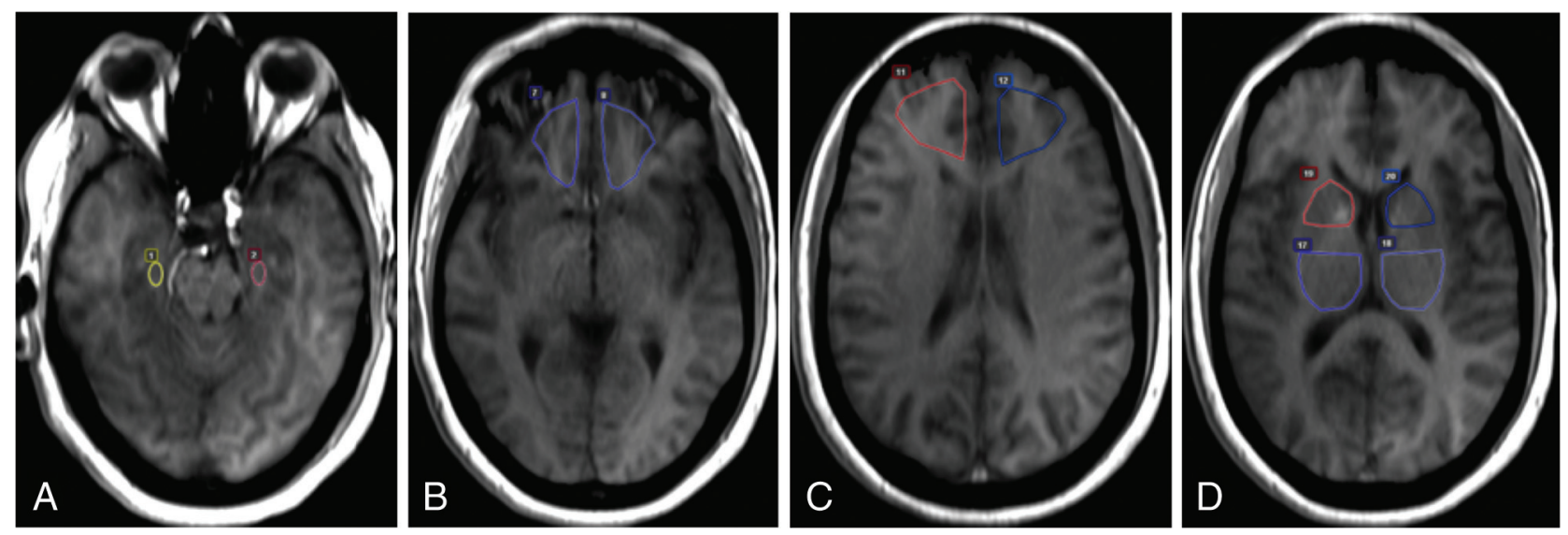

FIG 1. Illustration of ROI placement in a representative subject. ROIs were placed onto coregistered axial T1-weighted images in the following regions: hippocampus $(A)$, orbitofrontal $(B)$, prefrontal $(C)$, and anterior putamen/caudate and posterior putamen/thalamus $(D)$. Mirror ROls were placed for bilateral sampling. Region-specific DCE curves were generated.

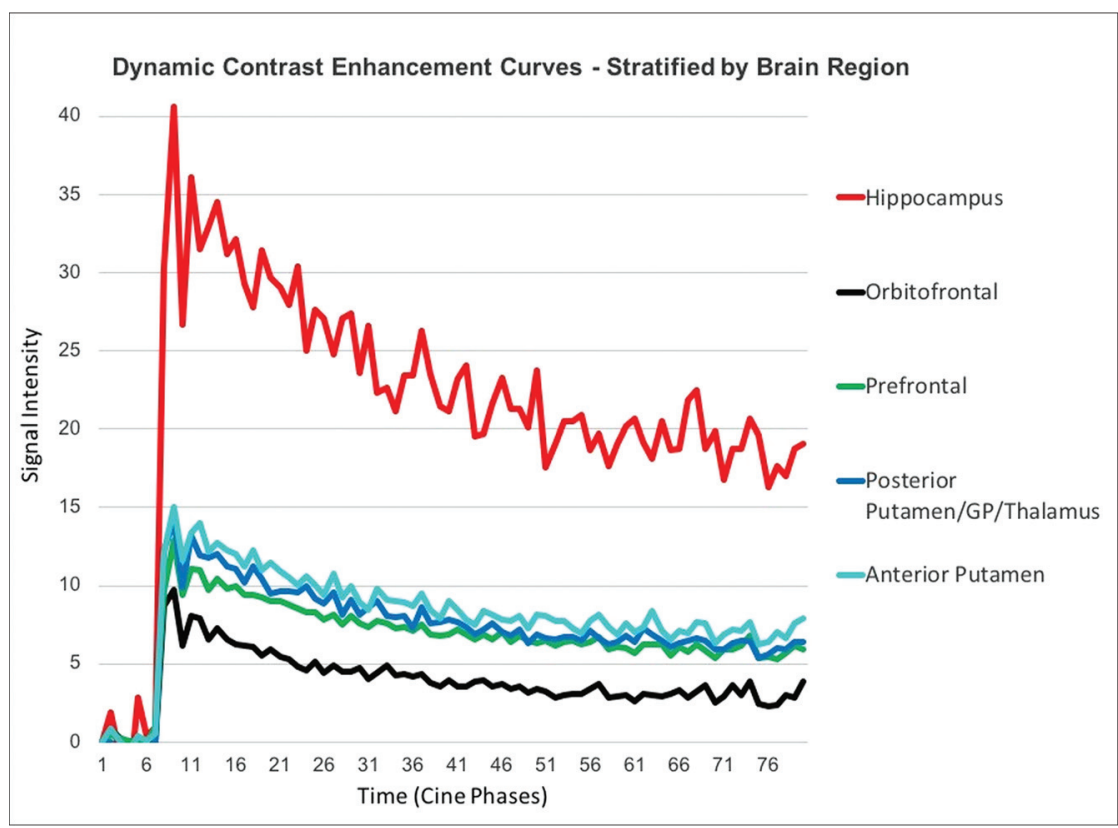

FIG 2. DCE curves in healthy control subjects, stratified by brain region. The hippocampi demonstrate statistically significantly higher areas under the curve compared with all other regions, indicating higher BBB permeability under normal conditions.

protocols. Whole-brain permeability imaging was performed using a DCE technique with 22-slice axial 3D spoiled gradient-recalled T1WI sequences at $0.5 \times 0.5 \times 5 \mathrm{~mm}$ resolution and 80 cine phases using $\mathrm{TR}=25 \mathrm{~ms}, \mathrm{TE}=3.8 \mathrm{~ms}, \mathrm{FOV}=24 \mathrm{~mm}$.

\section{Data Analysis}

Postprocessing into $K^{\text {trans }}(\mathrm{mL} / 100 \mathrm{~g} / \mathrm{min}), \mathrm{VE}(\mathrm{mL} / 100 \mathrm{~g})$, and $\mathrm{CBF}(\mathrm{mL} / 100 \mathrm{~g} / \mathrm{min}$ ) parametric maps was performed using Olea Sphere (Olea Medical, La Ciotat, France) with the Tofts extendedpermeability model by trained research personnel. The postprocessing technique was standardized with the arterial input function placed at the center of the cavernous internal carotid artery.

A standardized method was used for selective ROI placement by trained research personnel directly supervised by a board-certified neuroradiologist (with 19 years of experience). ROIs were placed onto coregistered axial T1-weighted images sampling the hippocam- pus, orbitofrontal and prefrontal regions, anterior putamen/caudate (including the globus pallidus and anterior limb of the internal capsule), and posterior putamen/ thalamus (including the globus pallidus and posterior limb of the internal capsule) (Fig 1), and meticulous care was taken not to include vascular structures, the choroid plexus, ventricles, CSF, and skull. ROIs with CBF values of $>100 \mathrm{~mL} / 100 \mathrm{~g} / \mathrm{min}$ were excluded from the statistical analysis to minimize contributions from vascular structures. ${ }^{8}$ Mirror ROIs were placed, and region-specific DCE curves were generated. The average size of the ROIs sampling each brain region was the following: hippocampus, $45 \mathrm{~mm}^{2}$; orbitofrontal, 454 $\mathrm{mm}^{2}$; prefrontal, $654 \mathrm{~mm}^{2}$; anterior puta$\mathrm{men} /$ caudate, $280 \mathrm{~mm}^{2}$; and posterior putamen/thalamus, $530 \mathrm{~mm}^{2}$.

\section{Statistical Analysis}

For statistical analysis, region-specific $K^{\text {trans }}, \mathrm{VE}$, and CBF means and SDs were computed. ANOVA was performed to determine statistical differences. The mean DCE curves for each brain region were generated. The area under the curve was computed in reference to the baseline before contrast arrival. The mean area under the curve was compared among brain regions using pair-wise $t$ test statistics. $P$ values $<.05$ were considered statistically significant.

\section{RESULTS}

Five subjects were included in the statistical analysis, with a total of 50 region-specific ROIs. One subject was excluded from statistical analysis due to motion degradation precluding postprocessing. The average age was $34.2 \pm 10.5$ years. All neuropsychological screening scores were within the normal range.

When we compared the generated mean DCE curves for each brain region across all subjects, the hippocampus demonstrated increased BBBP compared with all other regions (Fig 2). The mul- 
tiple pair-wise $t$ test comparisons revealed statistically significant higher areas under the curve for the hippocampus compared with the orbitofrontal $(P<.001)$ and prefrontal $(P<.001)$ regions, anterior putamen/caudate $(P<.002)$, and posterior putamen/ thalamus $(P<.001)$. This pattern was also observed across the individual subject data and was maintained even when brain regions were separated by right and left sides.

The model-based quantitative data in the hippocampus region yielded a mean $K^{\text {trans }}$ of $0.019 \pm 0.049$ and a mean VE of $0.008 \pm$ 0.016 , respectively. In contrast, the other sampled brain regions demonstrated a mean $K^{\text {trans }}$ of $0 \pm 0$ and mean VE of $0 \pm 0$ for both $K^{\text {trans }}$ and VE. ANOVA revealed a significantly increased BBBP in the hippocampal region compared with all other brain regions $(P=.03)$. Additionally, the hippocampi demonstrated the highest variability in quantitative data.

\section{DISCUSSION}

The BBB plays a critical role in limiting the entry of blood-derived toxins, pathogens, and cells into the brain. Our study investigated region-specific $\mathrm{BBB}$ permeability in healthy subjects, suggesting unique characteristics in the hippocampal region derived from the model-based quantitative analysis and area under the DCE curve. The DCE curve comprises 4 phases: 1 ) the initial rapid rise of the curve, depending on tissue perfusion flow rate; 2) the early peak, depending on tissue blood volume; 3 ) the downslope, depending on leakage into the interstitium; and 4) the later plateau phase, depending on the interstitial volume after return of the contrast agent into the blood compartment. ${ }^{9}$ Most important, the area under the DCE curve is affected by both perfusion (CBV, $\mathrm{CBF})$ and permeability $\left(K^{\text {trans }}, \mathrm{VE}\right)$ characteristics.

A review of the literature revealed that increased $B B B P$ in the hippocampus has been previously demonstrated with DCE-MR imaging in control subjects in a mouse model of BBB disruption, in which histologic assessment of BBB integrity served as the reference standard. ${ }^{7}$ Because the hippocampal microvasculature is supplied from both the anterior and posterior circulation, ${ }^{4}$ hypothetic explanations have related the increased susceptibility of the hippocampus to cerebrovascular autoregulatory dysfunction that has been similarly described in the sympathetic vascular innervation of the posterior circulation. ${ }^{10}$ Most important, there is molecular evidence for elevated expression of biomarkers of $\mathrm{BBB}$ disruption in the hippocampus. ${ }^{6}$

Another potential contributory factor is the concept of selective neuronal vulnerability of hippocampal neurons, which describes hippocampal neurons in the cornu ammonis 1 region as most sensitive to neurodegeneration cascades occurring in Alzheimer disease ${ }^{11}$ and demonstrating heightened sensitivity to oxidative stress, ${ }^{12}$ cerebral ischemia, ${ }^{13}$ and toxic-metabolic and inflammatory processes. ${ }^{14}$ This selective neuronal vulnerability can manifest in deficient DNA repair, calcium dysregulation, and glutamate hyperactivity in hippocampal neurons. ${ }^{15}$ Age-related hippocampal BBB breakdown has been demonstrated in individuals with mild cognitive impairment as well. ${ }^{16}$ In a mouse model of Alzheimer disease, microvascular impairment was found as a consequence of increased $\beta$ amyloid deposition. ${ }^{17}$ In patients with systemic lupus erythematosus, anti-DNA and anti- $N$-methyl-Daspartate-receptor antibodies crossing the BBB were associated with abnormal findings on hippocampal imaging and memory dysfunction. ${ }^{18}$ Furthermore, there is evidence that these antibodies were found to contribute to hippocampal neuronal apoptosis in patients with neuropsychiatric systemic lupus erythematosus. ${ }^{5,19}$ However, BBBP characteristics in the hippocampus compared with other distinct brain regions have not been previously described in healthy individuals, to our knowledge.

Even though our pilot study was adequately powered for the primary analysis, caution should be exercised not to overinterpret these results, given the small sample size demonstrating unique BBBP characteristics in the hippocampus. Given the challenge in avoiding choroid plexus contamination to obtain absolute ROI sampling of the hippocampal region, meticulous attention or innovative methods or both are warranted in further studies evaluating BBBP characteristics of the hippocampus in healthy and diseased subjects. Our findings are concordant with previous animal and human studies assessing hippocampal BBBP in neurodegenerative and neuroinflammatory diseases because increased baseline hippocampal vulnerability suggests that BBB disruption in the hippocampus may potentially contribute to early pathophysiologic disease manifestations. Most important, larger cohort studies in healthy individuals are needed to further substantiate these findings.

\section{CONCLUSIONS}

We evaluated region-specific DCE-MR imaging-derived BBBP in healthy subjects, which suggests unique BBBP characteristics in the hippocampus, concordant with prior animal studies. This work may help further our understanding of specific brain regions susceptible to neurologic diseases affecting the BBB. Larger scale prospective studies evaluating hippocampal BBBP characteristics are needed to confirm these findings and potentially incorporate them into diagnostic and therapeutic strategies.

Disclosures: Jana Ivanidze-UNRELATED: Employment: Weill Cornell Medical College, Comments: Assistant Professor; Grants/Grants Pending: American Society of Neuroradiology Research and Education Foundation, Comments: American Society of Neuroradiology Research Scholar Award. Angela Hoang-RELATED: Grant: National Institutes of Health.* Joan M. Chi-RELATED: Grant: National Institutes of Health, Comments: research grant 1P01AI073693.* Katherine Cheng-RELATED: Grant: National Institutes of Health/National Institute of Allergy and Infectious Diseases protocol 1P01A1073693.* Bruce Volpe-RELATED: Grant: National Institutes of Health program project grant.* Betty Diamond_RELATED: Grant: National Institutes of Health*; UNRELATED: Grants/Grants Pending: National Institutes of Health.* *Money paid to the institution.

\section{REFERENCES}

1. Bartels AL, Kortekaas R, Bart J, et al. Blood-brain barrier P-glycoprotein function decreases in specific brain regions with aging: a possible role in progressive neurodegeneration. Neurobiol Aging 2009;30:1818-24 CrossRef Medline

2. Loeffler C, Dietz K, Schleich A, et al. Immune surveillance of the normal human CNS takes place in dependence of the locoregional blood-brain barrier configuration and is mainly performed by CD3(+)/CD8(+) lymphocytes. Neuropathology 2011;31:230-38 CrossRef Medline

3. Jeneson A, Squire LR. Working memory, long-term memory, and medial temporal lobe function. Learn Mem 2012;19:15-25 CrossRef Medline

4. Tatu L, Vuillier F. Structure and vascularization of the human hippocampus. Front Neurol Neurosci 2014;34:18-25 CrossRef Medline

5. Mackay M, Tang CC, Volpe BT, et al. Brain metabolism and auto- 
antibody titres predict functional impairment in systemic lupus erythematosus. Lupus Sci Med 2015;2:e000074 CrossRef Medline

6. Ueno M, Chiba Y, Murakami R, et al. Blood-brain barrier and blood-cerebrospinal fluid barrier in normal and pathological conditions. Brain Tumor Pathol 2016;33:89-96 CrossRef Medline

7. Weidman EK, Foley CP, Kallas O, et al. Evaluating permeability surface-area product as a measure of blood-brain barrier permeability in a murine model. AJNR Am J Neuroradiol 2016;37:1267-74 CrossRef Medline

8. Sanelli PC, Ugorec I, Johnson CE, et al. Using quantitative CT perfusion for evaluation of delayed cerebral ischemia following aneurysmal subarachnoid hemorrhage. AJNR Am J Neuroradiol 2011;32: 2047-53 CrossRef Medline

9. Cuenod CA, Balvay D. Perfusion and vascular permeability: basic concepts and measurement in DCE-CT and DCE-MRI. Diagn Interv Imaging 2013;94:1187-204 CrossRef Medline

10. Sanelli PC, Jacobs MA, Ougorets I, et al. Posterior reversible encephalopathy syndrome on computed tomography perfusion in a patient on "Triple H" therapy. Neurocrit Care 2005;3:46-50 CrossRef Medline

11. Hyman BT, Van Hoesen GW, Damasio AR, et al. Alzheimer's disease: cell-specific pathology isolates the hippocampal formation. Science 1984;225:1168-70 CrossRef Medline

12. Wang $\mathrm{X}, \mathrm{Pal} \mathrm{R}, \mathrm{Chen} \mathrm{XW}$, et al. High intrinsic oxidative stress may underlie selective vulnerability of the hippocampal CA1 region. Brain Res Mol Brain Res 2005;140:120-26 CrossRef Medline

13. Olsson T, Wieloch T, Smith ML. Brain damage in a mouse model of global cerebral ischemia. Effect of NMDA receptor blockade. Brain Res 2003;982:260-69 CrossRef Medline

14. Bartsch T, Döhring J, Reuter S, et al. Selective neuronal vulnerability of human hippocampal CA1 neurons: lesion evolution, temporal course, and pattern of hippocampal damage in diffusion-weighted MR imaging. J Cereb Blood Flow Metab 2015;35:1836-45 CrossRef Medline

15. Wang $X$, Michaelis EK. Selective neuronal vulnerability to oxidative stress in the brain. Front Aging Neurosci 2010;2:12 CrossRef Medline

16. Montagne A, Barnes SR, Sweeney MD, et al. Blood-brain barrier breakdown in the aging human hippocampus. Neuron 2015;85: 296-302 CrossRef Medline

17. Dorr A, Sahota B, Chinta LV, et al. Amyloid- $\beta$-dependent compromise of microvascular structure and function in a model of Alzheimer's disease. Brain 2012;135:3039-50 CrossRef Medline

18. Gulinello M, Wen J, Putterman C. Neuropsychiatric symptoms in lupus. Psychiatr Ann 2012;42:322-28 CrossRef Medline

19. Lauvsnes MB, Beyer MK, Kvaloy JT, et al. Association of hippocampal atrophy with cerebrospinal fluid antibodies against the NR2 subtype of the N-methyl-D-aspartate receptor in patients with systemic lupus erythematosus and patients with primary Sjögren's syndrome. Arthritis Rheumatol 2014;66:3387-94 CrossRef Medline 